Rev. Int. Contam. Ambie. 34 (4) 725-735, 2018

DOI: 10.20937/RICA.2018.34.04.13

\title{
EVALUACIÓN DEL EFECTO DEL CADMIO SOBRE LA ACTIVIDAD DE FENOLOXIDASAS DE TEJIDO CALLOSO DE Epithelantha micromeris INOCULADO CON RIZOBACTERIAS
}

\author{
Analí TOLEDO SALAS ${ }^{1}$, María Victoria HERNÁNDEZ PIMENTEL ${ }^{1}$, \\ Aída Verónica RODRÍGUEZ TOVAR ${ }^{2}$, Leonor Angélica GUERRERO ZÚÑIGA ${ }^{3}$ \\ y Angélica RODRÍGUEZ DORANTES ${ }^{1 *}$
}

${ }^{1}$ Laboratorio de Fisiología Vegetal, Departamento de Botánica, Escuela Nacional de Ciencias Biológicas, Instituto Politécnico Nacional, Prolongación Carpio y Plan de Ayala s/n, 11340 Ciudad de México, México

${ }^{2}$ Departamento de Microbiología, Escuela Nacional de Ciencias Biológicas, Instituto Politécnico Nacional, Prolongación Carpio y Plan de Ayala s/n, 11340 Ciudad de México, México

${ }^{3}$ Dirección de Investigación en Transformación de Hidrocarburos, Gerencia de Transformación de Biomasa, Instituto Mexicano del Petróleo, Eje Central Lázaro Cárdenas 152, San Bartolo Atepehuacan, 07730 Ciudad de México, México

*Autora para correspondencia: rodorantes@yahoo.com.mx

(Recibido junio de 2017; aceptado febrero 2018)

Palabras clave: metales pesados, cultivo in vitro, peroxidasas, hemoperoxidasas, respuesta antioxidante

\section{RESUMEN}

Se propone el proceso de biotización como una nueva técnica para el cultivo de tejidos vegetales que permite la asociación entre plantas y microorganismos en condiciones in vitro. A la inoculación de tejido calloso cultivado in vitro se le denomina bacterización, proceso en que las bacterias estimulan el crecimiento de las plántulas e inducen cambios en su desarrollo, lo que les permite potenciar sus mecanismos de respuesta ante el estrés. Uno de los efectos más notables en las plantas expuestas a cadmio es el incremento o disminución de las enzimas antioxidantes, con la posible participación de las fenoloxidasas. El presente trabajo evaluó la respuesta de cultivos de callo de Epithelantha micromeris (Engelm.) inoculados con rizobacterias promotoras del crecimiento vegetal, a través de la actividad de guaiacol peroxidasas y hemoperoxidasas como respuesta al estrés oxidativo producido por la exposición a cadmio. De los resultados obtenidos, la presencia de las rizobacterias per se ocasionó una condición de estrés; no obstante, en presencia de cadmio se indujo una respuesta antioxidante que incluyó el incremento de fenoloxidasas, indicadoras de estrés oxidativo.

Key words: heavy metals, in vitro culture, peroxidases, hemoperoxidases, antioxidant response

\begin{abstract}
A biotization process is proposed as a new technique in plant tissue culture that allows in vitro the association between plants and microorganisms. Bacterization is the inoculation of callus tissue cultivated in vitro. In this process, bacteria promote the plants growth, induce changes in their development and increase the response mechanisms against stress conditions. One of the most notable effects of plants exposed to cadmium is the increment or inhibition of antioxidant enzymes, with the possible participation of phenoloxidases. The present study evaluated the response of callus tissue of Epithelantha micromeris
\end{abstract}


(Engelm.) inoculated with plant growth promoting rhizobacteria through guaiacol peroxidases and hemoperoxidases activity as oxidative stress responses produced by cadmium exposition. From the results obtained, rhizobacteria occurrence per se generated a stress condition; however, in the presence of cadmium an antioxidant response was induced that includes the increment of phenoloxidases, which are oxidative stress indicators.

\section{INTRODUCCIÓN}

El cadmio $(\mathrm{Cd})$, uno de los elementos más tóxicos que se encuentran en el aire, el agua y el suelo. Es considerado como un metal no esencial sin ninguna función fisiológica conocida; sin embargo, induce cambios complejos en las plantas a nivel genético, bioquímico y fisiológico que conducen a la fitotoxicidad, debido a que se absorbe y se distribuye rápidamente a través del sistema vascular (del Castillo 2012). Algunos de los efectos más notables en las plantas expuestas a cadmio son aumento en los niveles de la peroxidación lipídica, estrés oxidativo y cambios en el incremento o disminución de las enzimas antioxidantes, además de reducción de la absorción de nutrientes esenciales, con la posible participación de las fenoloxidasas en el tratamiento de metales pesados. Estas enzimas se han empleado para fitomonitorear áreas industriales y densamente urbanizadas por su sensibilidad a la contaminación atmosférica, lo que las clasifica como otro de los biomarcadores clásicos. Dentro de este grupo de enzimas se encuentran las guaiacol peroxidasas y hemoperoxidasas, que pertenecen a una gran familia multigénica por su gran número de isoformas, así como por la regulación heterogénea de su expresión; están involucradas en un amplio número de procesos fisiológicos de las plantas, en particular durante las primeras fases de su crecimiento, lo que incluye condiciones de estrés (Escalante et al. 2012). Las rizobacterias conocidas en la literatura con el acrónimo PGPR (del inglés plant growth promoting rhizobacteria) son bacterias benéficas que se presentan como una alternativa a los fertilizantes químicos y plaguicidas (Kloepper y Beauchamp 1992). Estas poblaciones microbianas rizosféricas son capaces de ejercer efectos específicos sobre el crecimiento vegetal, tales como producción de fitohormonas, disolución y mineralización de fosfatos, fijación asimbiótica del nitrógeno atmosférico y producción de sideróforos y antibióticos (van Loon et al. 1998, Vessey 2003). La inoculación de plantas con este tipo de microorganismos ha emergido recientemente como una estrategia rizorremediadora para potenciar su crecimiento y con ello favorecer su tolerancia al estrés producido por metales pesados (Epelde et al. 2010, Koo et al. 2010, Ma et al. 2011, Rajkumar et al. 2012, 2013). Las técnicas de cultivo de células y tejidos vegetales que consideran el crecimiento de células, tejidos u órganos vegetales in vitro, en condiciones asépticas, controladas y libres de microorganismos (Street 1977, Ferl y Paul 2000), permiten la disección de los sistemas complejos de las plantas; en particular, el cultivo de callo constituye un sistema adecuado para evaluar la actividad biológica de agroquímicos, toxinas microbianas y aleloquímicos, así como la tolerancia a metales pesados y los procesos de detoxificación en plantas que han reportado autores como Mumma y Davidonis (1983), Hogan y Manners (1990), Harms (1992), Song et al. (1994), Zacchini et al. (2003), Golan-Goldhirsh et al. (2004), Azevedo et al. (2005a), Bittsánsky et al. (2005), Gullner et al. (2005), Nehnevajova et al. (2007), Herrera et al. (2008) y Shekhawat et al. (2010).

El efecto benéfico de la asociación entre plantas y microorganismos en condiciones in vitro se propone como una nueva técnica para el cultivo de tejidos vegetales, que corresponde al proceso de biotización (Sharma et al. 2015). A la inoculación de explantes o tejido calloso cultivados in vitro se le denomina bacterización, proceso en que las bacterias estimulan el crecimiento de las plántulas e inducen cambios en su desarrollo, lo que les permite potenciar sus mecanismos de respuesta ante el estrés. Los beneficios de la bacterización tanto in vitro como ex vitro dependen de la especie vegetal, la forma de cultivo y las condiciones de crecimiento (Lazarovits y Nowak 1997, Pillay y Nowak 1997, Nowak 1998). Epithelantha micromeris (Engelm.) F.A.C. Weber ex Britt. \& Rose es una cactácea apreciada por coleccionistas nacionales y extranjeros por su morfología como planta de ornato. Se distribuye en el desierto de Chihuahua, desde el oeste de Texas y Nuevo México en EUA hasta el norte de México en los estados de Coahuila, Chihuahua, Nuevo León y San Luís Potosí, donde están presentes variantes endémicas (VillavicencioGutiérrez et al. 2012). También se ha empleado como modelo de cultivo in vitro para estudio de la 
interacción con PGPR y en la tolerancia y remoción de metales pesados (Golan-Goldhirsh et al. 2004, Corona-Álvarez et al. 2017).

El presente trabajo evaluó la respuesta del crecimiento de cultivos de callo de Epithelantha micromeris (Engelm.) inoculados con rizobacterias promotoras del crecimiento vegetal y la actividad de guaiacol peroxidasas y hemoperoxidasas como respuesta al estrés oxidativo producido por la exposición a cadmio.

\section{MATERIAL Y MÉTODOS}

\section{Origen de las rizobacterias empleadas como inoculantes}

Las rizobacterias empleadas como inoculantes son cepas productoras de sideróforos y fitohormonas, aisladas por Avilés-Narváez (2016): Serratia sp. A (Rz A), de la rizósfera de Sphaeralcea angustifolia (Cav.) G. Don; Serratia sp. L (Rz L), y Serratia sp. F (Rz F), de la rizosfera de Tithonia diversitifolia (Hemsl.) A. Gray. Todas estas especies crecen en un sitio contaminado con metales pesados (As, $\mathrm{Cu}, \mathrm{Pb}$ y Zn) localizado en Villa de la Paz, San Luís Potosí, México $\left(23^{\circ} 41^{\prime} \mathrm{N}, 100^{\circ} 42^{\prime} \mathrm{O}\right)$, región que ha sido objeto de explotación minera intensa durante varias décadas (Vásquez et al. 2006).

\section{Mantenimiento y propagación del tejido calloso de $E$. micromeris}

El tejido calloso empleado de E. micromeris, se obtuvo a partir del material fresco del cultivo in vitro de plantas de la colección biológica del Laboratorio de Fisiología Vegetal de la Escuela Nacional de Ciencias Biológicas, IPN. Su propagación se realizó a partir de tejido calloso fresco no diferenciado que se mantuvo en medio sólido 37 (Murashige-Skoog [MS] 1/4 sales) (Murashige y Skoog 1962) con la adición de $0.5 \mathrm{mg} / \mathrm{L}$ de ácido indolacético (AIA) y $1 \mathrm{mg} / \mathrm{L}$ de bencil amino purina (BAP).

\section{Establecimiento de los bioensayos}

Se fraccionaron callos de E. micromeris de $1 \mathrm{~cm}^{2}$ y se depositaron cuatro fracciones de $0.25 \mathrm{~cm}^{2}$ en frascos de cultivo tipo Gerber con tapas Magenta, que contenían $30 \mathrm{~mL}$ de medio sólido 37 (MS 1/4 sales) con $0.5 \mathrm{mg} / \mathrm{L}$ de AIA y $1 \mathrm{mg} / \mathrm{L}$ de BAP, a los que se les adicionó cadmio $\left(3 \mathrm{CdSO}_{4} \cdot 8 \mathrm{H}_{2} \mathrm{O}\right)$ en tres concentraciones: $0.1,0.5$ y $1.0 \mathrm{mM}$, inoculados por separado con las tres rizobacterias seleccionadas. La preparación de los inóculos bacterianos se realizó según lo establecido por Ramírez-Melo et al. (2013) a partir de un cultivo masivo en placa con medio sólido LB (Luria-Bertani) cultivado por $48 \mathrm{~h}$, del que se tomó una asada y se ajustó una suspensión bacteriana en agua destilada estéril a una densidad de $5 \times 10^{7}$ células $/ \mathrm{mL}$. De esta suspensión bacteriana se tomaron $0.25 \mathrm{~mL}$ para inocular las masas celulares, de acuerdo con lo reportado por Corona-Álvarez et al. (2017). Los tratamientos experimentales fueron los siguientes: testigo (sólo con medio MS 37), $\mathrm{MS}+\mathrm{Cd}$ $0.1 \mathrm{mM}, \mathrm{MS}+\mathrm{Cd} 0.5 \mathrm{mM}$ y MS + Cd $1.0 \mathrm{mM}$; con rizobacterias: MS+ Rz A, MS + Rz L y MS+Rz F; y el inoculante y las tres concentraciones de cadmio: $\mathrm{MS}+\mathrm{Rz} \mathrm{A}+\mathrm{Cd} 0.1 \mathrm{mM}, \mathrm{MS}+\mathrm{Rz} \mathrm{A}+\mathrm{Cd} 0.5 \mathrm{mM}$, $\mathrm{MS}+\mathrm{Rz} \mathrm{A}+\mathrm{Cd} 1.0 \mathrm{mM}, \mathrm{MS}+\mathrm{Rz} \mathrm{L}+\mathrm{Cd} 0.1 \mathrm{mM}$. $\mathrm{MS}+\mathrm{Rz} \mathrm{L}+\mathrm{Cd} 0.5 \mathrm{mM}, \mathrm{MS}+\mathrm{Rz} \mathrm{L}+\mathrm{Cd} 1.0 \mathrm{mM}$, $\mathrm{MS}+\mathrm{Rz} \mathrm{F}+\mathrm{Cd} 0.1 \mathrm{mM}, \mathrm{MS}+\mathrm{Rz} \mathrm{F}+\mathrm{Cd} 0.5 \mathrm{mM}$ $\mathrm{y} \mathrm{MS}+\mathrm{Rz} \mathrm{F}+\mathrm{Cd} 1.0 \mathrm{mM}$. Los tratamientos se instalaron por triplicado y los frascos se dejaron en una cámara de cultivo en condiciones de iluminación continua con una lámpara de luz fría de 4500 lux $\left(67.5 \mu \mathrm{mol} / \mathrm{m}^{2} / \mathrm{s}\right)$ a temperatura ambiente $\left(28^{\circ} \mathrm{C}\right) \mathrm{y}$ con fotoperiodo de $16: 8$ por ocho días.

\section{Evaluación del crecimiento y viabilidad del tejido calloso de $E$. micromeris}

Después del tiempo de incubación, las fracciones de callo se analizaron visualmente para evaluar algún daño debido a las condiciones experimentales o por el efecto de la inoculación. De acuerdo con el criterio de Souissi y Kremer (1998), se tomaron en cuenta el color y la reducción del crecimiento o la desintegración del tejido calloso. Estos elementos se calificaron en una escala de 0 a 4: 0: sin decoloración, cambios visibles ni reducción en el crecimiento; 1 : con ligera decoloración y sin reducción visible del crecimiento; 2: con cambios en el color del tejido y ligera reducción del crecimiento; 3 : con cambios en el color del tejido, reducción obvia del crecimiento y contracciones del tejido frecuentes, y 4: cambios en el color del tejido, desintegración del callo y reducción severa del crecimiento.

Se obtuvo el peso fresco de las masas celulares y se evaluó la viabilidad celular de éstas, con el empleo del colorante azul de Evans, que no es permeable y produce baja toxicidad. El empleo de este colorante esta descrito por varios autores (Gaff y Okong'oOgola 1971, Turner y Novacky 1974, Souissi y Kremer 1994, 1998, Song et al. 1999, Iriti et al. 2006, Kollárová et al. 2017) para determinar el número relativo de células muertas en cultivos de células en suspensión y de callo, mediante la cuenta de células muertas que se tiñen con el azul y el número de células vivas que no presentan esta coloración. La tinción 
se realizó de acuerdo con el método modificado por Toledo (2012) y consistió en colocar las masas celulares en tubos de ensaye, adicionando $1 \mathrm{~mL}$ de solución de azul de Evans al $0.5 \%$ durante 10 min. Transcurrido ese tiempo se decantó el líquido de cada muestra y se enjuagó con agua destilada hasta que la solución decantada fuera incolora. Se homogenizaron los fragmentos de callo con un homogeneizador de tejidos Potter-Elvehjem (PTFE, Sigma-Aldrich) y se les adiciono $1 \mathrm{~mL}$ de agua destilada. Las muestras se centrifugaron a $3000 \mathrm{rpm}$ durante $3 \mathrm{~min}$ a temperatura ambiente, se recuperó el sobrenadante en tubos de ensaye de $5 \mathrm{~mL}$ para, finalmente, leer su absorbancia a una longitud de onda de $630 \mathrm{~nm}$. La concentración final de azul de Evans se determinó con el empleo de una curva tipo de este colorante.

Para todas las masas celulares obtenidas, se determinó el contenido total de proteína por la técnica convencional de Bradford (1976), tomando $100 \mathrm{mg}$ de callo que se homogenizaron con el Potter-Elvehjem (PTFE, Sigma-Aldrich) con la adición de un regulador de fosfatos $(50 \mathrm{mM}, \mathrm{pH}=6.0$, en frío). Una vez obtenidos los extractos celulares se tomó $1 \mathrm{~mL}$ de éstos y se le adicionaron $3 \mathrm{~mL}$ del reactivo azul de Coomassie G-250. Se dejó incubar por 10 min a temperatura ambiente y se leyó la absorbancia del complejo colorido formado a $595 \mathrm{~nm}$. Se empleó una curva tipo de albúmina bovina para la determinación de la concentración de proteína. La concentración de proteína total celular se expresó como $\mathrm{mg} / \mathrm{mL}$ de proteína celular total/g de peso fresco.

\section{Evaluación de la actividad de fenoloxidasas como respuesta al estrés oxidativo del tejido calloso de E. micromeris expuesto a cadmio}

La actividad de las guaiacol peroxidasas (GPX) de cada extracto celular se determinó por el método espectrofotométrico de la oxidación de guaiacol de Guerrero-Zúñiga y Rodríguez-Dorantes (2009), depositando lo siguiente en tubos Eppendorf de $1.5 \mathrm{~mL}$ : $100 \mu \mathrm{L}$ del extracto celular obtenido $+1 \mathrm{~mL}$ de regulador de fosfato de potasio $(100 \mathrm{mM}, \mathrm{pH}=6.0)$ $+32 \mu \mathrm{L}$ de guaiacol $0.2 \mathrm{M}+32 \mu \mathrm{L}$ de peróxido de hidrógeno $0.03 \mathrm{M}$. Los tubos se incubaron por $30 \mathrm{~min}$ a temperatura ambiente hasta obtener evidencia de la actividad de guaiacol peroxidasas a través del desarrollo de una coloración café-rojiza, característica de la oxidación y polimerización del guaiacol; finalmente, se leyó la absorbancia a $436 \mathrm{~nm}$. La actividad de hemoperoxidasas (HPX) de los extractos celulares se determinó con el método espectrofotométrico de la oxidación de tetrametilbencidina (TMBZ), de acuerdo con Herrera et al. (2008), mediante el depósito de los siguientes componentes en tubos Eppendorf de $1.5 \mathrm{~mL}: 100 \mu \mathrm{L}$ de muestra $+1 \mathrm{~mL}$ de regulador de acetato de sodio $(0.20 \mathrm{M}, \mathrm{pH}=5.3)+100 \mu \mathrm{L}$ de $\mathrm{TMBZ}+50 \mu \mathrm{L}$ de peróxido de hidrógeno al $0.17 \%$. Los tubos se incubaron por $30 \mathrm{~min}$ a temperatura ambiente y se evidenció la actividad de hemoperoxidasas a través del desarrollo de la coloración azul característica de la oxidación de tetrametilbencidina. Finalmente se leyó la absorbancia a $652 \mathrm{~nm}$. La actividad enzimática específica de ambas enzimas se calculó empleando la ecuación modificada por Escalante et al. (2012):

$\mathrm{C}=\frac{\mathrm{As}}{\xi} \times \frac{\mathrm{R}}{\mathrm{S}} \times \frac{1}{\mathrm{t}}$

donde As es la absorbancia obtenida de las lecturas a 436 y $652 \mathrm{~nm}$; $\xi$ el coeficiente de extinción molar del guaiacol $\left(\varepsilon_{436}=6400 \mathrm{M} / \mathrm{cm}\right)$ y de la TMBZ $\left(\varepsilon_{652}\right.$ $=39000 \mathrm{M} / \mathrm{cm}$ ); R el volumen total del ensayo; $\mathrm{S}$ el volumen de la muestra, y t el tiempo de incubación de la reacción enzimática. La actividad de ambas enzimas se expresó como nM de guaiacol oxidado/ $\mathrm{min} / \mathrm{g}$ de peso fresco y $\mathrm{nM}$ de TMBZ oxidada $/ \mathrm{min} / \mathrm{g}$ de peso fresco.

A todos los resultados obtenidos se les aplicó un análisis de varianza (ANOVA) y prueba de TukeyKramer de comparación múltiple, empleando el paquete estadístico GraphPad InStat, v. 3.10 (2009). Se realizó un análisis numérico comparativo entre las diferentes condiciones experimentales con los datos de viabilidad celular (porcentaje de azul de Evans), proteína total y actividad enzimática (GPX y HPX), empleando el programa NTSyS-PC (numerical taxonomy and multivariate analysis system) $\mathrm{v}$. 2.11X (2005), para la aplicación del índice de distancia euclidiana. Una vez obtenidos estos valores, se construyó el fenograma respectivo utilizando el método de agrupamiento UPGMA (unweighted pair group method of arithmetic averages) y se estableció la veracidad y confianza del fenograma obtenido a través de la obtención del coeficiente de correlación producto-momento de Pearson.

\section{RESULTADOS Y DISCUSIÓN}

\section{Evaluación de la viabilidad celular y el crecimien- to del tejido calloso de $E$. micromeris}

El crecimiento de las masas celulares, relacionado con el contenido de proteína total cuantificado, mostró que en general los tratamientos inoculados no lo promovieron. Los valores de dicho crecimiento fueron de 1 a $12 \mathrm{mg} / \mathrm{mL}$ de proteína celular total $/ \mathrm{g}$ 
peso fresco (Fig. 1a), en comparación con los tratamientos en presencia de $\mathrm{Cd}(12 \mathrm{a} 45 \mathrm{mg} / \mathrm{mL}$ proteína celular total/g peso fresco), en los que no se observó un efecto dañino sobre el tejido calloso de E. micromeris en las tres concentraciones de este metal. Por el contrario, se observó un efecto promotor del crecimiento (Fig. 1b). Se sugiere que esta respuesta puede relacionarse con un efecto hormético producido por el Cd (inducción de crecimiento ante un gradiente de concentraciones del metal), pues se ha determinado que como primera barrera a la toxicidad de este metal, la mayoría de las especies acumulan $\mathrm{Cd}$, con el fin de limitar su transporte (Vásquez et al. 2006).

Considerando que en la viabilidad celular determinada (Fig. 2) un mayor porcentaje cuantificado del colorante corresponde a una elevada muerte celular en el tejido calloso, éste correspondió a los tratamientos del tejido calloso de E. micromeris inoculados con 5 y $8 \%$ de azul de Evans, seguidos por el tratamiento con el medio de cultivo con $3 \%$. Los tratamientos que presentaron un menor indicio de muerte celular fueron los que tenían la presencia del inoculante y Cd con alrededor del $2 \%$. Souissi y Kremer (1998) mencionan que la presencia de rizobacterias produce un efecto promotor o inhibidor del crecimiento de los callos. En este estudio, la inoculación del tejido calloso de E. micromeris ocasionó un efecto fitotóxico no solamente en la disminución del tamaño de los callos $(\mathrm{MS}+\mathrm{Rz} \mathrm{A}=0.11 \mathrm{~g}, \mathrm{MS}+\mathrm{Rz} \mathrm{L}=0.07 \mathrm{~g}$ y MS + Rz $\mathrm{F}=0.087 \mathrm{~g}$ ), en comparación con los tratamientos con el medio $(0.18 \mathrm{~g})$, sino también en su apariencia (Fig. 3), ya que las masas celulares presentaron una coloración obscura, característica de un proceso de necrosis en el callo. Según el criterio de Souissi y Kremer (1998), esto se considera un daño de nivel 3 (cambios en la coloración, reducción obvia en el crecimiento y contracción de los callos). Estos autores y Goodman et al. (1986) sugieren que la variación en los síntomas ocasionados por las rizobacterias proviene de diferencias en el modo de acción y la bioquímica de la interacción planta-rizobacteria en el tejido calloso.

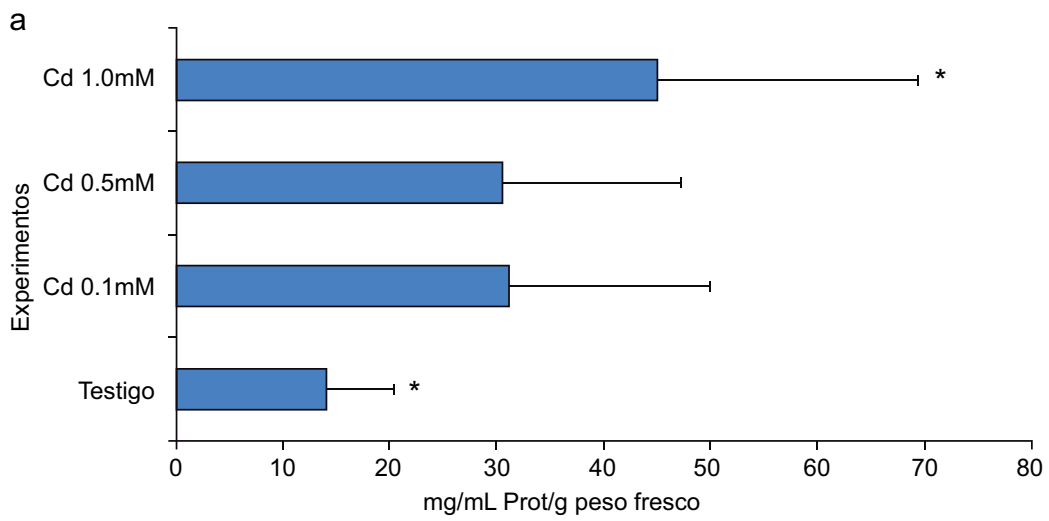

b

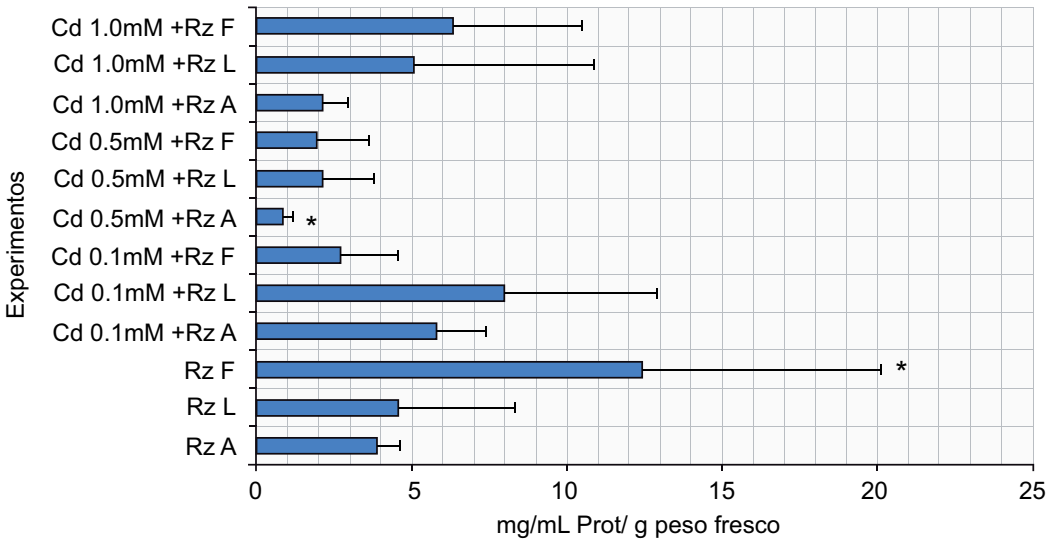

Fig. 1. Cuantificación de proteína total celular del tejido calloso de E. micromeris: (a) exposición a cadmio; (b) exposición a cadmio e inoculación con rizobacterias. Sólo las condiciones con marcadas con un asterisco $(*)$ resultaron estadísticamente significativas $(\mathrm{n}=12, \mathrm{p}<0.001)$ 


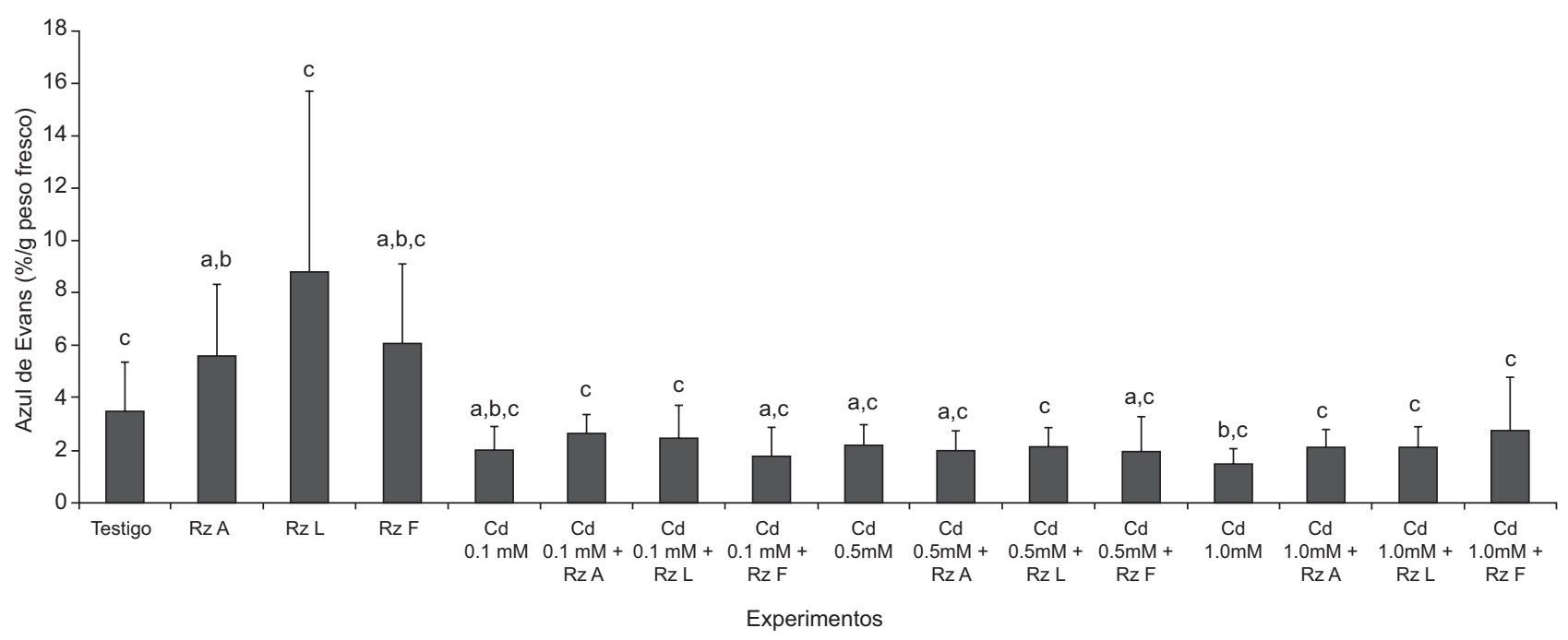

Fig. 2. Evaluación de la viabilidad del tejido calloso de E. micromeris en todas las condiciones experimentales probadas. Las diferentes letras muestran diferencias estadísticamente significativas $(\mathrm{n}=12, \mathrm{p}<0.001)$

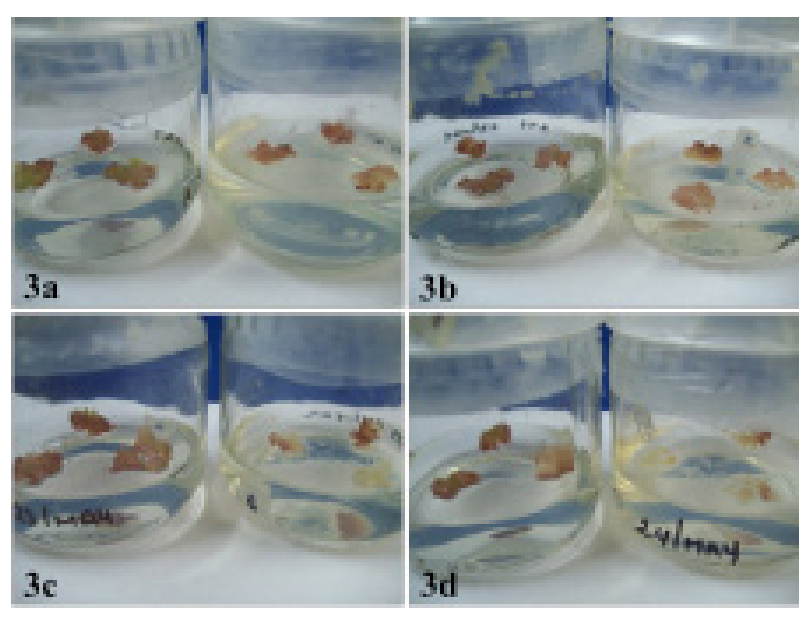

Fig. 3. Desarrollo de los cultivos de tejido calloso de E. micromeris inoculados con Rz L: (a) tratamiento con $\mathrm{Rz} \mathrm{L}$, (b) tratamiento con $\mathrm{Cd} 0.1 \mathrm{mM}+\mathrm{Rz} \mathrm{L}$, (c) tratamiento con Cd $0.5 \mathrm{mM}+\mathrm{Rz} \mathrm{L}$ y Cd $1.0 \mathrm{mM}+\mathrm{Rz} \mathrm{L}$

\section{Evaluación de la actividad de GPX y HPX ante la presencia de $\mathrm{Cd}$ en el tejido calloso de $E$. $m i$ - cromeris}

La actividad obtenida de las GPX fue mayor en los tratamientos con $\mathrm{Cd}(0.1,0.5$ y $1.0 \mathrm{mM})$ comparada con el tratamiento MS (Fig. 4a), entre los 80 y $110 \times 10^{6} \mathrm{nM}$ de guaiacol oxidado/g peso fresco. En los tratamientos de los callos inoculados (Fig. 4b), la actividad de GPX obtenida fue menor (entre 6 y $46 \times 10^{6} \mathrm{nM}$ de guaiacol oxidado/g peso fresco); en particular la presencia de $\mathrm{Cd}$ y las rizobacterias $\mathrm{Rz} \mathrm{L}$ y $\mathrm{Rz} F$, indujeron una mayor actividad de estas enzimas. La actividad obtenida de las HPX contrastó con la de las GPX, ya que en las primeras ésta fue menor en las masas celulares con el tratamiento MS y la presencia de $\mathrm{Cd}$ (Fig. 4c) (de 2 a $8 \times 10^{6} \mathrm{nM} \mathrm{TMBZ} / \mathrm{min} / \mathrm{g}$ peso fresco) y mayor en los tratamientos inoculados (Fig. 4d) (de 24 a $39 \times 10^{6} \mathrm{nM} \mathrm{TMBZ} / \mathrm{min} / \mathrm{g}$ peso fresco).

La respuesta de la Rz L y Rz F se repitió en presencia de las diferentes concentraciones de $\mathrm{Cd}$. La actividad de HPX, que fue mayor en los cultivos de callo inoculados con la Rz A, disminuyó con la adición del metal pesado de 38 a $24 \times 10^{6} \mathrm{nM}$ TMBZ/ $\mathrm{min} / \mathrm{g}$ peso fresco. Uno de los parámetros bioquímicos reportados como biomarcadores de respuesta a la exposición a metales pesados en las plantas es el incremento o inhibición de la actividad de enzimas antioxidantes. Pernía et al. (2008) y Rodríguez et al. (2008) reportan que la presencia de cadmio produce un exceso de especies reactivas de oxigeno (ROS) que se asocia con incremento en la actividad de este tipo de fenoloxidasas. En este trabajo se obtuvo un incremento en la actividad de HPX, la cual podría vincularse con este tipo de respuestas, donde las condiciones de estrés generaron una reacción protectora ante las condiciones de toxicidad. En términos de la actividad de fenoloxidasas, ésta disminuyó en todos los tratamientos probados al aumentar la concentración de $\mathrm{Cd}$, lo cual coincidió con lo reportado por Szöllösi et al. (2009). Esto sugiere la presencia de un evento asociado a la dosis-respuesta en los tratamientos inoculados, lo que no se observó en presencia del metal solo con un incremento en la respuesta oxidativa a sus altas concentraciones. 

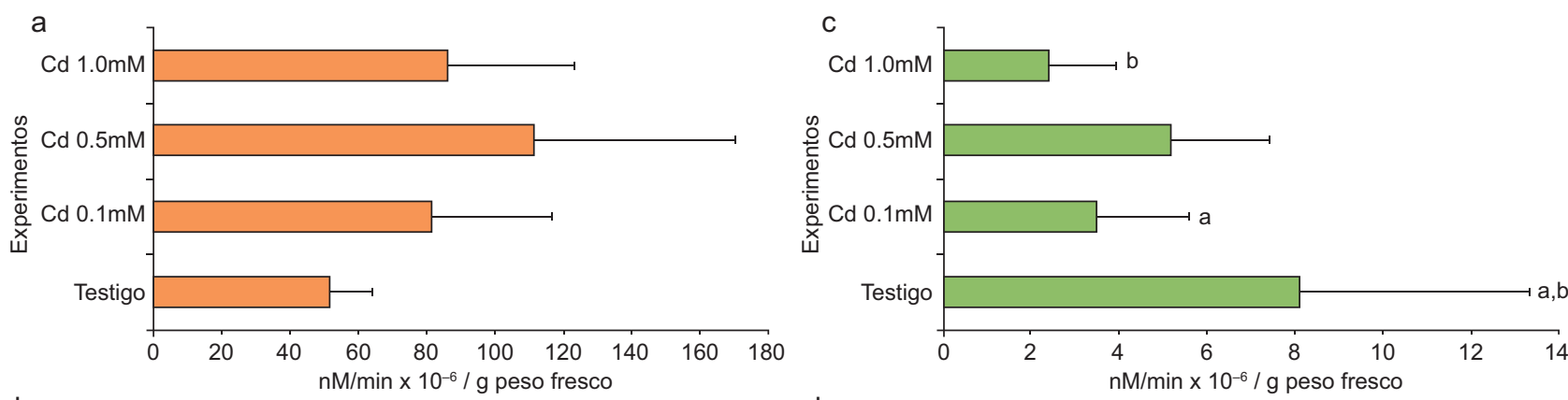

b

d
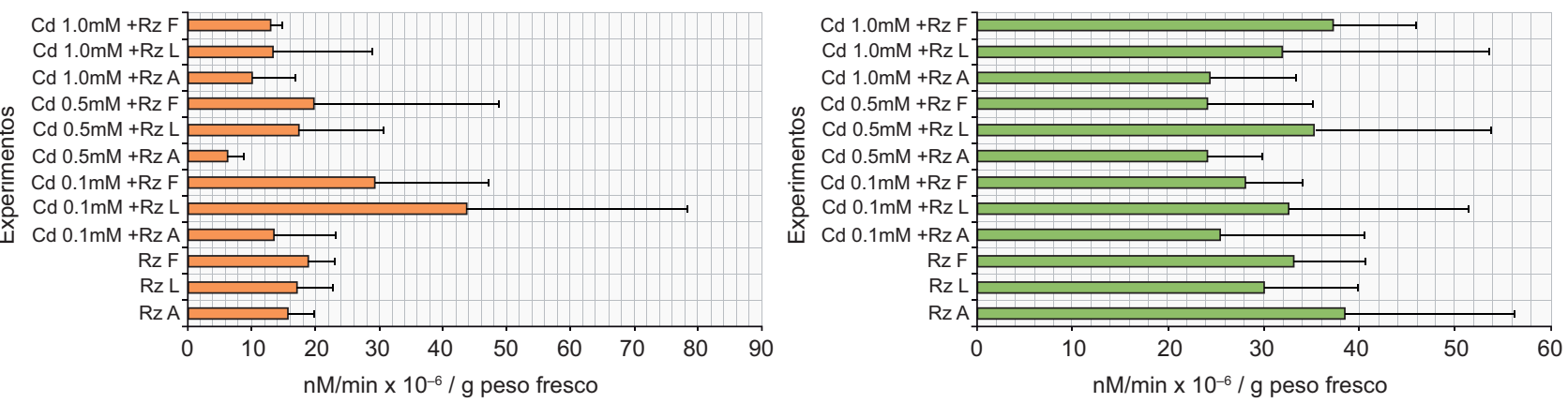

Fig. 4. Evaluación de la actividad enzimática del tejido calloso de E. micromeris: (a) actividad de GPX de callos expuestos a cadmio, (b) actividad de GPX de callos expuestos a cadmio e inoculados con rizobacterias, (c) actividad de HPX de callos expuestos a cadmio y (d) actividad de HPX de callos expuestos a cadmio e inoculados con las tres rizobacterias. Las cifras con diferente letra resultaron estadísticamente significativas $(\mathrm{n}=12, \mathrm{p}<0.001)$

\section{Respuesta integral del tejido calloso de $E$. micro- meris ante la presencia de $\mathrm{Cd}$ y los inoculantes}

La figura 5 muestra la asociación de la respuesta integral del tejido calloso ante la presencia de $\mathrm{Cd}$ y las tres rizobacterias. El fenograma muestra la asociación inicial del Grupo I, conformado por dos conjuntos bien definidos: uno que asocia las respuestas particulares del tejido calloso ante la presencia de cadmio, donde los resultados que contribuyen a esta agrupación son la proteína total y la actividad de GPX; y otro conformado por el resto de las condiciones experimentales, que a su vez constituyen el Grupo II. En éste se muestra la respuesta particular del tejido calloso crecido en condiciones normales, relacionado con el Grupo IIa, en el cual se incluyen todas las condiciones experimentales con la inoculación del tejido calloso. En este grupo se incluyen conjuntos de condiciones muy específicas: las rizobacterias Serratia sp. L y Serratia sp. F, cuyas respuestas son comunes aun en agrupaciones separadas. En este sentido, destacan las respuestas obtenidas en las condiciones experimentales con la adición de $0.1 \mathrm{mM}$ de $\mathrm{Cd}$ y la presencia de las rizobacterias mencionadas. De todas las agrupaciones, la respuesta particular de Serratia sp. A fue similar con la exposición de los callos a 0.5 y $1.0 \mathrm{mM}$ de Cd. Iori et al. (2012) reportan que los cultivos de callo de dos clones parentales de Populus nigra L. (Poli y 58-861) expuestos a sulfato de cadmio a las concentraciones de 0.15 y $0.25 \mathrm{mM}$, tuvieron respuestas de crecimiento y actividad de GPX diferentes. En el clon Poli, la actividad disminuyó a la concentración de $0.15 \mathrm{mM}$, y en ambos clones esto ocurrió con $0.25 \mathrm{mM}$ de $\mathrm{Cd}$. Los autores reportan que esto pudo deberse al efecto producido por la unión del $\mathrm{Cd}$ a los grupos funcionales enzimáticos, ya que según Prasad (1995) y Joshi y Mohanty (2004), éste interfiere con el doblamiento y las propiedades catalíticas enzimáticas. Azevedo et al. (2005a, b) evaluaron el efecto del cadmio sobre los sistemas enzimáticos de defensa en tejido calloso de Helianthus annuus L., en particular aquellos que están involucrados en prevenir el estrés oxidativo. El tejido calloso creció en presencia de $\mathrm{Cd}$ a las concentraciones de $0,0.005,0.050$ y $0.50 \mathrm{mM}$, y se observó una reducción significativa del contenido de proteína total del tejido calloso a las concentraciones de 0.050 y $0.50 \mathrm{mM}$. Por su parte, la actividad de peroxidasas se incrementó en los callos expuestos a $0.005 \mathrm{mM}$ y decreció en aquellos expuestos a la concentración de $0.50 \mathrm{mM}$. Los autores mencionan que este decremento pudo deberse al hecho de que se presenta una alteración en la integración de la 


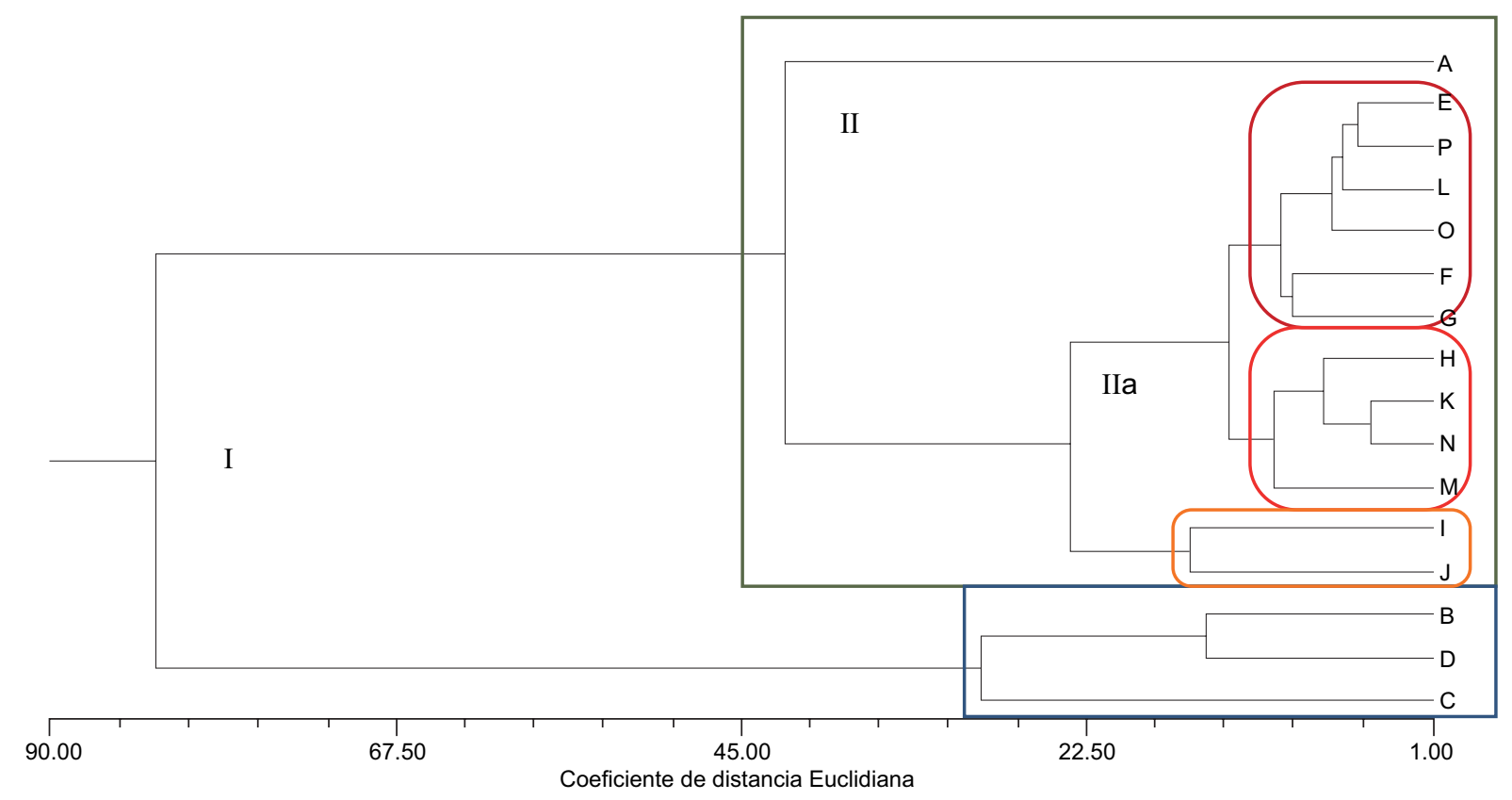

Fig. 5. Fenograma del agrupamiento de las condiciones experimentales de los cultivos de callo de E. micromeris $(\mathrm{r}=0.90)$; A: testigo; B: Cd 0.1 mM; C: Cd 0.5 mM; D: Cd 1.0 mM; E: Rz A; F: Rz L; G: Rz F; H: Cd 0.1 mM + Rz A; I: Cd 0.1 $\mathrm{mM}+\mathrm{Rz} \mathrm{L} ; \mathrm{J}: \mathrm{Cd} 0.1 \mathrm{mM}+\mathrm{Rz}$ F; K: Cd $0.5 \mathrm{mM}+\mathrm{Rz}$ A; L: Cd 0.5 mM + Rz L; M: Cd 0.5 mM + Rz F; N: Cd 1.0 $\mathrm{mM}+\mathrm{Rz} \mathrm{A}$; O: Cd 1.0 mM + Rz L, y P: Cd $1.0 \mathrm{mM}+\mathrm{Rz} \mathrm{F}$

membrana plasmática, lo cual sugiere que los sistemas del callo desarrollan mecanismos antioxidantes que les permiten responder al estrés provocado por el Cd.

Los resultados aquí reportados y los que se citan de la literatura, concluyen que este tipo de respuestas en tejido calloso están relacionadas con la sensibilidad al cadmio y sobre todo con la posibilidad de potenciar mecanismos antioxidantes que impliquen sistemas de defensa relacionados con las actividades enzimáticas. Esto sugiere la presencia de mecanismos celulares que proporcionan tolerancia al metal y, con ello, permiten la adaptación. Dichos mecanismos celulares también pueden vincularse con la inducción de sistemas enzimáticos como respuesta al estrés oxidativo. Fornazier et al. (2002), Shützendübel y Polle (2002) y Benavides et al. (2005) reportan que la modulación de la actividad enzimática por $\mathrm{Cd}$ depende fuertemente de las concentraciones de cadmio, el tiempo de exposición a éste y de la especie u órgano vegetal.

\section{CONCLUSIONES}

Las actividades inducibles de guaiacol peroxidasas y hemoperoxidasas son indicadores de estrés oxidativo generado por la presencia de cadmio, el cual se incrementó en los tratamientos inoculados con las tres rizobacterias empleadas y que, en conjunto con la respuesta antioxidante, confirieron un efecto protector al tejido calloso de E. micromeris.

\section{AGRADECIMIENTOS}

Se agradece a la Secretaría de Investigación y Posgrado del Instituto Politécnico Nacional el apoyo financiero otorgado para la realización de este trabajo a través del Proyecto SIP: 20121516. Los autores agradecen a la Comisión de Operaciones y Fomento de Actividades Académicas (COFAA-IPN), al EDI (Estímulo al Desempeño de InvestigadoresIPN) y al Sistema Nacional de Investigadores (SNICONACyT).

\section{REFERENCIAS}

Avilés-Narváez L. (2016). Caracterización fisiológica de rizobacterias aisladas de plantas metaloacumuladoras con potencial rizoremediador. Tesis de Licenciatura, Escuela Nacional de Ciencias Biológicas, Instituto Politécnico Nacional, México, 78 pp. 
Azevedo H., Pinto C.G.G. y Santos C. (2005a). Cadmium effects in sunflower: Nutritional imbalances in plants and calluses. J. Plant Nutr. 28(12), 2221-2231.

DOI: $10.1080 / 01904160500324808$

Azevedo H., Pinto C.G.G. y Santos C. (2005b). Cadmium effects in sunflower: Membrane permeability and changes in catalase and peroxidase activity in leaves and calluses. J. Plant Nutr. 28(12), 2233-2241.

DOI: $10.1080 / 01904160500324816$

Benavides M.P., Gallego S.M. y Tomaro M.L. (2005). Cadmium toxicity in plants. Braz. J. Plant Physiol. 17(1), 21-34. DOI: 10.1590/S1677-04202005000100003

Bittsánsky A., Kömives T., Gullner G., Gyulai G., Kiss J., Heszky L., Radimszky L. y Rennemberg H. (2005). Ability of transgenic poplars with elevated glutathione content to tolerate zinc(2+) stress. Environ. Int. 31(2), 251-254. DOI: 10.1016/j.envint.2004.10.001

Bradford M. (1976). Rapid and sensitive method for the quantitation of microgram quantities of protein utilizing the principle of protein-dye binding. Anal. Biochem. 72(1-2), 248-254. DOI: 10.1016/0003-2697(76)90527-3

Corona-Álvarez D., Guerrero-Zúñiga L.A. y RodríguezDorantes A. (2017). Effect of phytohormones and rhizobacteria on Epithelantha micromeris callus growth. Int. J. Curr. Microbiol. App. Sci. 6(7), 1002-1009. DOI: 10.20546/ijcmas.2017.607.121

Del Castillo G.I. (2012). Estudio del efecto antioxidante de las ficobiliproteínas en presencia de cadmio como agente de estrés oxidative sobre la germinación de Axonopus affinis, Lactuca sativa y Lens esculenta. Tesis de Licenciatura. Escuela Nacional de Ciencias Biológicas, Instituto Politécnico Nacional, México, $112 \mathrm{pp}$.

Epelde L., Becerril J.M., Barrutia O., González-Oreja J.A. y Garbisu C. (2010). Interactions between plant and rhizosphere microbial communities in a metalliferous soil. Environ. Pollut.158(5), 1576-1583.

DOI: 10.1016/j.envpol.2009.12.013

Escalante S.C., Rodríguez D.A., Vásquez M.S., Rodríguez T.A., Guerrero Z.L.A., Pérez N.O., Franco M.O. y Ponce M. (2012). Evaluación del efecto de cadmio sobre la germinación y elongación radical de semillas bacterizadas de Axonopus affinis y Festuca rubra. Polibotanica. 34, 185-201.

Ferl R. y Paul A.L. (2000). Genome organization and expression. En: Biochemistry and molecular biology of plants (Buchanan B.B, Gruissem W. Jones R.L., Eds.). American Society of Plant Physiologists, EUA, pp. 312-357.

Fornazier R.F., Ferreira R.R., Pereira G.J.G., Molina S.M.G., Smith J.R., Lea P.J. y Azevedo R.A. (2002). Cadmium stress in sugar cane callus cultures: effect on antioxidant enzymes. Plant Cell Tiss. Org. 71(2), 125-131.

DOI: $10.1023 / \mathrm{A}: 1019917705111$

Gaff D.F. y Okong'o-Ogola O. (1971). The use of nonpermeating pigments for testing the survival of cells. J. Exp. Bot. 22(3), 756-758. DOI: 10.1093/jxb/22.3.756

Golan-Goldhirsh L.A., Barazani O., Nepovim A., Soudek P., Smrcek S., Dufkova L., Krenkova S., Yrjala K., Schröder P. y Vanek T. (2004). Plant response to heavy metals and organic pollutants in cell culture and at whole plant. J. Soil Sediment. 4(2), 133-140.

DOI: 10.1007/BF02991058

Goodman R.N., Kiraly Z. y Wood K.R. (1986). The biochemistry and physiology of plant disease. University of Missouri Press, Columbia, MO.

Guerrero-Zúñiga L.A. y Rodríguez-Dorantes A. (2009). Efecto de la presencia de fenantreno sobre la expresión de proteínas y la actividad enzimática radical de Cyperus hermaphroditus. Polibotánica 27, 103-130.

Gullner G, Kömives T. y Rennemberg H. (2005). Enhanced tolerance of transgenic poplar plants overexpressing gglutamylcysteine synthetase towards chloroacetanilide herbicides. J. Exp. Bot. 52(358), 971-979.

DOI: $10.1093 /$ jexbot $/ 52.358 .971$

Harms H.H. (1992). In vitro systems for studying phytotoxicity and metabolic fate of pesticides and xenobiotics in plants. Pestic. Sci. 35(3), 277-281.

DOI: $10.1002 / p s .2780350313$

Herrera G.M., Rodríguez D.A.M. y Guerrero Z.L.A. (2008). Evaluación del crecimiento, actividad de hemoperoxidasas y remoción de fenantreno de los cultivos celulares de Fouquieria splendens y Fouquieria fasciculata. Polibotánica 25, 101-119.

Hogan M.E. y Manners G.D. (1990). Allelopathy of small everlasting (Antennaria microphylla): Phytotoxicity to leafy spurge (Euphorbia esula) in tissue culture. J. Chem. Ecol. 16(3), 931-939.

DOI: 10.1007/BF01016501

Iori V., Pietrini F., Massacci A. y Zacchini M. (2012). Induction of metal binding compounds and antioxidative defence in callus cultures of two black poplar (Populus nigra) clones with different tolerance to cadmium. Plant Cell Tiss. Org. 108(1), 17-26.

DOI: $10.1007 / \mathrm{s} 11240-011-0006-8$

Iriti M., Sironi M., Gomarasca S., Casazza A.P., Soave C. y Faoro F. (2006). Cell death-mediated antiviral effect of chitosan in tobacco. Plant Physiol. Biochem. 44(11-12), 893-900.

DOI: $10.1016 /$ j.plaphy.2006.10.009

Joshi M.K. y Mohanty P. (2004). Chlorophyll a fluorescence as a probe of heavy metal ion toxicity in plants. En: Chlorophyll a fluorescence: A signature of photosynthesis. Advances in photosynthesis and 
respiration (Papageorgiou G.C. y Govindjee, Eds.), vol. 19. Springer, Dordrecht, pp 637-661.

DOI: 10.1007/978-1-4020-3218-9 25

Kloepper J. y Beauchamp Ch. (1992). A review of issues related to measuring colonization of plant roots by bacteria. Can. J. Microbiol. 38(12), 1219-1232. DOI: 10.1139/m92-202

Kollárová K., Vatehová Z., Kučerová D. y Lišková D. (2017). Cadmium impact, accumulation and detection in poplar callus cells. Environ. Sci. Pollut. Res. 24(18), 5340-15346. DOI: 10.1007/s11356-017-9158-3

Koo S.Y., Hong S.H., Ryu H.W. y Cho K. (2010). Plant growth-promoting trait of rhizobacteria isolated from soil contaminated with petroleum and heavy metals. J. Microbiol. Biotechn. 20(3), 587-593.

Lazarovits G. y Nowak J. (1997). Rhizobacteria for improvement of plant growth and establishment. Hort. Science. 32(2), 188-192.

Ma Y., Prasad M.N.V., Rajkumar M. y Freitas H. (2011). Plant growth promoting rhizobacteria and endophytes accelerate phytoremediation of metalliferous soils. Biotechnol. Adv. 29(2), 248-258.

DOI: 10.1016/j.biotechadv.2010.12.001

Mumma R.O. y Davidonis G.H. (1983). Plant tissue culture and pesticide metabolism. En: Progress in Pesticide Biochemistry and Toxicology. (Huston D.H. y Roberts T.R. Eds.). John Wiley \& Sons, New York, pp. 255-280.

Murashige T. y Skoog A. (1962). A revised medium of rapid growth and bioassays with tobacco tissue. Physiol. Plantarum 15(3), 473-497. DOI: 10.1111/j.1399-3054.1962.tb08052.x

Nehnevajova E., Herzig R., Erismann K.H. y Schwitzguébel J.P. (2007). In vitro breeding of Brassica juncea L. to enhance metal accumulation and extraction properties. Plant Cell Rep. 26(4), 429-437.

DOI: $10.1007 / \mathrm{s} 00299-006-0264-9$

Nowak J. (1998). Benefits of in vitro "biotization" of plant tissue cultures with microbial inoculants. In Vitro Cell. Dev. Biol. Plant. 34(2), 122-130.

DOI: $10.1007 / \mathrm{BF} 02822776$

Pernia B., de Souza A., Reyes R. y Castrillo M. (2008). Biomarcadores de contaminación por cadmio en las plantas. Interciencia 3(2), 112-119.

Pillay V.K. y Nowak J. (1997). Inoculum density, temperature and genotype effects on epiphytic and endophytic colonization and in vitro growth promotion of tomato (Lycopersicon esculentum L.) by a pseudomonad bacterium. Can. J. Microbiol. 43(4), 354-361. DOI: 10.1139/m97-049

Prasad M.N.V. (1995). Cadmium toxicity and tolerance in vascular plants. Environ. Exp. Bot. 25(4), 525-545. DOI: $10.1016 / 0098-8472(95) 00024-0$
Rajkumar M., Sandhya S., Prasad M.N.V. y Freitas H. (2012). Perspectives of plant associated microbes in heavy metal phytoremediation. Biotechnol. Adv. 30(6), 1562-1574. DOI: 10.1016/j.biotechadv.2012.04.011

Rajkumar M., Prasad M.N.V., Sandhya S. y Freitas H. (2013). Climate change driven plant-metal-microbe interactions. Environ. Int. 53(1), 74-86.

DOI: 0.1016/j.envint.2012.12.009

Ramírez-Melo M., Ruiz-Flores N., Vásquez-Murrieta S., Rodríguez-Tovar A., Guerrero-Zúñiga A. y RodríguezDorantes A. (2013). Plant growth promotion and protecting effect to heavy metals of rhizobacteria on inoculated Lens esculenta seeds. Int. J. AgriScience 3(5), 414-422.

Rodríguez S.M., Martínez C.N., Romero P.M.C., del Río L.A. y Sandalio L.M. (2008). Toxicidad del cadmio en plantas. Ecosistemas 17(3), 139-146.

Sharma V., Kamal B., Srivastava N., Negi Y., Dobriyal A.K. y Jadon S.V. (2015). Enhancement of in vitro growth of Swertia chirayita Roxb. Ex Fleming cocultured with plant growth promoting rhizobacteria. Plant Cell Tiss. Org. 121(1), 215-225.

DOI: $10.1007 / \mathrm{s} 11240-014-0696-9$

Shekhawat G.S., Verma K., Jana S., Singh K., Teotia P. y Prasad A. (2010). In vitro biochemical evaluation of cadmium tolerance mechanism in callus and seedlings of Brassica juncea. Protoplasma 239(1-4), 31-38. DOI: 10.1007/s00709-009-0079-y

Shützendübel A. y Polle A. (2002). Plant responses to abiotic stresses: Heavy metal-induced oxidative stress and protection by mycorrhization. J. Exp. Bot. 53(372), 1351-1365.

Song H.S., Lim S.M. y Widholm J.M. (1994). Selection and regeneration of soybeans resistant to the pathotoxic culture filtrates of Septoria glycines. Phytopathology 84(9), 948-951. DOI: 10.1094/Phyto-84-948

Song J., Kanazawa I., Sun K., Murata T. y Yokoyama K.K. (1999). Color coding the cell death status of plant suspension cells. BioTechniques 26(6), 1060-1062.

Souissi T. y Kremer R.J. (1994). Leafy spurge (Euphorbia esula) cell cultures for screening deleterious rhizobacteria. Weed Sci. 42(2), 310-315.

Souissi T. y Kremer R.J. (1998). A rapid microplate callus bioassay for assessment of rhizobacteria for biocontrol of leafy spurge (Euphorbia esula L.). Biocontrol Sci. Techn. 8(1), 83-92. DOI: 10.1080/09583159830450

Street H.E. (1977). Cell (suspension) cultures techniques. En: Plant tissue and cell culture (Street H.E., Ed.). Blackwell Scientific Publishing, Oxford, Reino Unido, pp. 61-102.

Szöllösi R., Vargas I.S., Erdei L.y Mihalik E. (2009). Cadmium-induced oxidative stress and antioxidative mechanisms in germinating Indian mustard (Brassica 
juncea L.) seeds. Ecotox. Environ. Safe. 72(5), 13371342. DOI: 10.1016/j.ecoenv.2009.04.005

Toledo S.A. (2012). Evaluación de la respuesta antioxidativa de tejido calloso de Epithelantha micromeris expuesto a un metal pesado e inoculado con rizobacterias. Tesis de Licenciatura, Escuela Nacional de Ciencias Biológicas, Instituto Politécnico Nacional, México, 123 pp.

Turner J.G. y Novacky A. (1974). The quantitative relation between plant and bacterial cells involved in the hypersensitive reaction. Phytopathology 64(1), 885890. DOI: 10.1094/Phyto-64-885

Van Loon L.C., Bakker P. y Pieterse C.M.J. (1998). Systemic resistance induced by rhizosphere bacteria. Annu. Rev. Phytopathol. 36(1), 453-483.

DOI: 10.1146/annurev.phyto.36.1.453

Vásquez M.S., Migueles G.I., Franco H.O., Govaerts B. y Dendooven L. (2006). C and N mineralization and microbial biomass in heavy metal-contaminated soil. Eur. J. Soil Biol. 42(2), 89-98.

DOI: 10.1016/j.ejsobi.2005.10.002
Vessey K. (2003). Plant growth promoting rhizobacteria as biofertilizers. Plant Soil 255(2), 571-586.

DOI: 10.1023/A:1026037216893

Villavicencio-Gutiérrez E.E., González-Cortés A. y Carranza-Pérez M.A. (2012). Micropropagación de Epithelantha micromeris (Engelm.) F.A.C. Weber ex Britt. \& Rose cactácea ornamental y recurso fitogenético del desierto chihuahuense. Rev. Mex. Cien. For. 3(14), 83-102.

Zacchini M., Rea E., Tullio M. y de Agazio M. (2003). Increased antioxidative capacity in maize calli during and after oxidative stress induced by a long lead treatment. Plant Physiol. Biochem. 41(1), 49-54.

DOI: $10.1016 / \mathrm{S} 0981-9428(02) 00008-6$ 